\title{
Assortative sexual mixing patterns in male-female and male- male partnerships in Melbourne, Australia: implications for HIV and STI transmission
}

\author{
Eric P.F. Chow ${ }^{1,2}$, Tim R.H. Read ${ }^{1,2}$, Matthew G. Law ${ }^{3}$, Marcus Y Chen ${ }^{1,2}$, Catriona S Bradshaw ${ }^{1,2}$, \\ Christopher K. Fairley ${ }^{1,2}$
}

1. Melbourne Sexual Health Centre, Alfred Health, Melbourne, VIC, Australia

2. Central Clinical School, Faculty of Medicine, Nursing and Health Sciences, Monash University, Melbourne, VIC, Australia 3. The Kirby Institute, UNSW Australia, Sydney, NSW, Australia

\section{Introduction}

Assortative mixing pattern have become a new and important focus in HIV/STI research in recent years.

Assortativity coefficient proposed by Newman is a measure of the correlations of characteristics in a network, and it has been used to assess the correlations of sexual practice in a sexual patterns recently.

$>$ Assortativity (like-with-like) mixing means individuals tend to mix with partners with similar risk practices (i.e. high number of sexual partners, and condomless sex); while disassortativity mixing means individuals tend to mix with partners with dissimilar risk practices.

$>$ Very limited data on mixing patterns on sexual practices, particularly in an Australian population.

\section{Aim}

$>$ To understand the assortative sexual mixing patterns on number of partners, and condom use in male-female and male-male partnerships attending a sexual health centre in Melbourne, Australia.

\section{Methods}

$>$ We included partners attended the Melbourne Sexual Health Centre (MSHC) on the same day between $9^{\text {th }}$ March 2011 and $31^{\text {st }}$ December 2014.

$>$ Partnerships were categorised into (1) male-female partnerships; or (2) male-male partnerships.

$>$ We excluded the partnership if one or both individuals met the following criteria: (1) aged <16 years; (2) HIV-positive; (3) sex workers; or (4) did not report number of partners.

$>$ Demographic characteristics (i.e. age) and sexual practices (i.e. number of partners and condom use in the last three months) were extracted.

$>$ In male-female partnerships, age and sexual practices were examined between men and women.

$>$ In male-male partnerships, each man in a partnership was randomly assigned to "Man 1" or "Man 2" by computer generated random sequences to ensure each man would have an equal chance of allocation to either group.

\section{Statistical Analyses}

D Spearman's rank correlation coefficient (rho) was calculated to examine the correlation of continuous variables (i.e. age and number of partners) between two individuals in a partnership.

$>$ Number of partners in the last three months was categorized into three groups: (1) 1 ; (2) $2-3$; and (4) $\geq 4$. Condom use was categorized as "always using condoms" and "not always using condoms".

$>$ The Newman's assortativity coefficient $(r)$ was used to measure assortative sexual mixing for number of partners and condom use.

$>$ The assortativity coefficient ranged from -1 to +1 , where +1 represents perfect assortativity, -1 represents perfect disassortativity, and 0 represents no assortativity.

$>$ Newman's assortativity coefficient $\geq 0.35$ indicates assortative, 0.260.34 indicates moderately assortative, and 0.15-0.25 indicates minimally assortative.

\section{Results}

$>1165$ male-female and 610 make-male partnerships were included.

$>$ Strong positive correlations in male-female partnerships (rho=0.709; $p<0.001)$ and male-male partnerships (rho $=0.553 ; p<0.001)$.

Figure 1. Correlation of age and number of partners in male-female and male-male partnerships

$80+$

${ }^{80}$

${ }^{70}-$
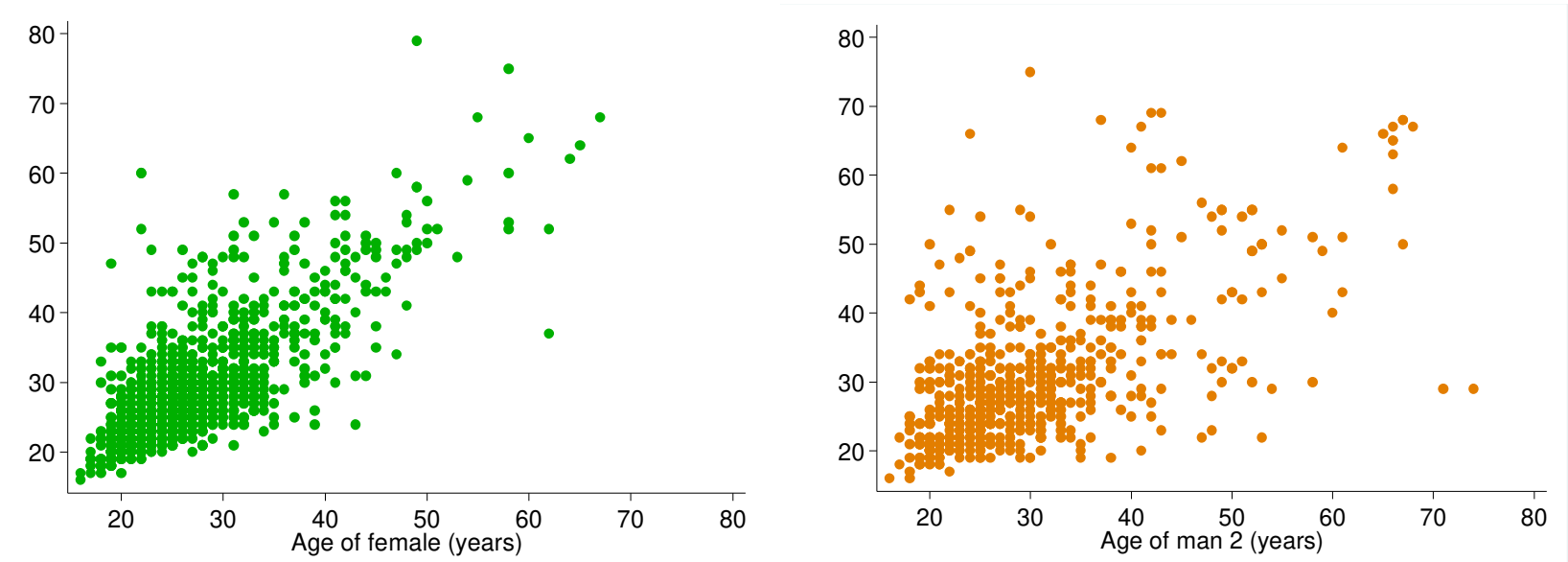

$>$ The assortativity coefficient for overall assortativity observed in number of partners was moderate in male-female partnerships partnership ( $r=0.255 ; 0.221$ to 0.289$)$ and in male-male partnerships $(r=0.264 ; 0.218$ to 0.309$)$.

$>$ There was a strong assortativity pattern of condom use in malefemale partnerships $(r=0.382 ; 0.353$ to 0.412$)$, and in male-male partnerships was observed ( $r=0.517,0.465$ to 0.569$)$.

Table 1. Newman's assortativity coefficient for assortativity by number of partners, and condom use in male-female and male-male partnerships.

\begin{tabular}{|l|c|c|c|c|}
\hline \multirow{2}{*}{ Year } & \multicolumn{2}{|c|}{ Male-female partnerships } & \multicolumn{2}{c|}{ Male-male partnerships } \\
\cline { 2 - 6 } & \# of partners & Condom use & \# of partners & Condom use \\
\hline \multirow{2}{*}{2011} & 0.239 & 0.381 & 0.291 & 0.553 \\
& $(0.160-0.319)$ & $(0.303-0.458)$ & $(0.176-0.406)$ & $(0.425-0.682)$ \\
2012 & 0.313 & 0.264 & 0.285 & 0.362 \\
& $(0.246-0.379)$ & $(0.200-0.327)$ & $(0.195-0.376)$ & $(0.256-0.469)$ \\
2013 & 0.227 & $0.300(0.248-$ & 0.259 & 0.565 \\
& $(0.165-0.289)$ & $0.353)$ & $(0.174-0.343)$ & $(0.466-0.664)$ \\
\multirow{2}{*}{2014} & 0.239 & $0.605(0.558-$ & 0.228 & 0.585 \\
& $(0.172-0.305)$ & $0.653)$ & $(0.145-0.310)$ & $(0.497-0.673)$ \\
\multirow{2}{*}{$2011-14$} & 0.255 & 0.382 & 0.264 & 0.517 \\
& $(0.221-0.289)$ & $(0.353-0.412)$ & $(0.218-0.309)$ & $(0.465-0.569)$ \\
\multirow{2}{*}{$p_{\text {trend }}$} & 0.718 & 0.722 & 0.034 & 0.628 \\
& & & & \\
\hline
\end{tabular}

\section{Conclusion}

$>$ Both male-female and male-male partnerships have a high assortativity sexual mixing pattern on age, number of partners, and condom use, that is, individuals are more likely to connect with partners with similar age and sexual practice.

$>$ The sexual mixing pattern is not purely assortative; and hence it may lead to increased HIV and STI transmission in certain risk groups.

\section{Contact Details}

Dr Eric PF Chow, PhD

Melbourne Sexual Health Centre

580 Swanston Street, Carlton, VIC 3053, Australia

Phone: +61 (3) 9341-6233

Email: Echow@mshc.org.au 